\title{
Nucleotide-Dependent Reprogramming of mRNAs Encoding Acetyl Coenzyme A Carboxylase and Lipoxygenase in Relation to the Fat Contents of Peanut
}

\author{
G. O. Osuji, T. K. Brown, and S. M. South \\ CARC Laboratories, Prairie View A\&M University, P.O. Box 519-2008, Prairie View, TX 77446, USA \\ Correspondence should be addressed to G. O. Osuji, goosuji@pvamu.edu
}

Received 18 February 2009; Revised 30 June 2009; Accepted 22 October 2009

Recommended by Bala Rathinasabapathi

\begin{abstract}
The nucleotide-dependent reprogramming of the mRNAs encoding acetyl coenzyme A carboxylase (ACC, EC 6.4.1.2) and lipoxygenase (LO, EC 1.13.11.12) was studied by Northern analysis. Equal concentrations of total RNA from the peanut (Arachis hypogaea L. cv. "Valencia") seedlings were probed with those glutamate dehydrogenase (GDH)-synthesized RNAs that were homologous to the mRNAs encoding ACC and LO. Treatment (4NTPs solution) of the peanuts increased the abundance of the mRNAs encoding the LO and ACC about 5 and 10 folds respectively but decreased the fat accumulation by $37 \%$ compared with the control peanut. Treatments (pyrimidine NTPs) of the peanut induced up to $60 \%$ more fat accumulation than the control. The high fat (40.0-44.0\%) contents of the UTP-, and 3NTP-treated peanuts were characterized by very high abundance of the mRNA for ACC, but low abundance of the mRNA for LO. The inverse relationship between the abundance of the mRNAs encoding ACC and LO compared with the seedlings fat contents was due to the plus/minus strands sequence homologies between the Northern probes for ACC and LO. Therefore, LO, ACC, and the GDH-synthesized RNAs participated in the regulation of the fat contents. These results could be useful in the environmental manipulation of the quantity and quality of peanut oil.
\end{abstract}

Copyright (C) 2009 G. O. Osuji et al. This is an open access article distributed under the Creative Commons Attribution License, which permits unrestricted use, distribution, and reproduction in any medium, provided the original work is properly cited.

\section{Introduction}

Acetyl coenzyme A carboxylase (ACC, EC 6.4.1.2) and lipoxygenase (LO, EC 1.13.11.12) are key enzymes in fatty acid metabolism $[1,2]$. ACC, which converts acetyl-CoA to manolyl-CoA, is the committed step of the fatty acid biosynthesis pathway. Two forms of ACC have been described in plants [3]. The first is a large protein similar to eukaryotictype enzyme, and is present in the cytosol. The second form is similar to the prokaryotic-type enzyme, and is present in the chloroplast [4]. Lipoxygenases are members of a large family of non-heme iron-containing dioxygenases that insert molecular oxygen into polyunsaturated fatty acids thereby converting them to hydroperoxide derivatives [5]. The hydroperoxides are reduced to hydroxyl fatty acids, or undergo enzymatic conversion to metabolites including lipoxins, leukotrienes [6], and free radical products [7]. Peanut seed may range in oil content from $44 \%$ to $56 \%$ with an average of $50 \%$ [8]. The oil contains $44.5 \%$ oleic acid,
32.3\% linoleic acid, and 13.9\% palmitic acid [9] and hence it has an excellent keeping quality. Therefore, it is expected that ACC and LOs would play important roles in determining the quantity and quality of fat storage reserves in the crop. Whereas the enzymic regulation of lipid metabolism has been studied [10], the redox-mediated reprogramming of the mRNAs encoding key enzymes has not been studied. Although the LOs catalyze the dioxygenation of polyunsaturated fatty acids, they have not been shown to regulate the accumulation of oil contents $[2,8]$. Improvements in the quality and quantity of the oil contents of oilseeds have traditionally been studied by breeding experiments [11-13], but the effects of the oxidative reprogramming of mRNA abundance on oil contents have not been studied. Current global production of peanut oil is $\sim 5$ million metric tons, and it is mainly used for food, although there is potential application for biodiesel production [14]. The quality of vegetable oil is important in determining its grade as biodiesel [15]. Peanut is an unpredictable legume oilseed 
crop and its yield is influenced by complex environmental factors [16]. Therefore, an understanding of the oxidative stress factors in conjunction with breeding program [17] might permit the development of a rapid method for increasing the fat content of the crop.

Plant growth, differentiation, and biomass accumulation are regulated by glutamate dehydrogenase [18, 19]. The enzyme synthesizes some RNA [20] as it isomerizes in response to changes in the plant's environment [21]. When plants were synchronously subjected to a wide range of redox stress factors, including toxic metal salts [22, 23], herbicides [24], pesticides [25], fertilizer nutrients [26], acid soils [20], pathogens [27], and nucleotides, their GDH isoenzyme populations fluctuated between zero and the highest number of 28 isoenzymes [28]. The ordered responses of plant metabolism to wide range of redox stress factors based on the isomerization of the plant's GDH (GDH methods) have been applied to investigate the coordinate metabolic regulation of serially harvested alfalfa $[20,22]$, and the regulation of cellulosic biomass accumulation [29]. Redox stress-dependent genomic studies are therefore factorially exhaustive and metabolically comprehensive compared with traditional studies on randomized multidimensional exogenous conditions that did not define the endogenous limits and impacts of the redox potentials of the treatments [30]. It is important to analyze the redox stress factors that limit agricultural productivity because the results could unveil possible synergistic and/or antagonistic interactions [31].

In the analysis of oxidative stress-dependent reprogramming of peanut mRNAs, it was established that the GDHsynthesized RNAs coordinately regulated catabolism and anabolism [29]. Also, by inversely regulating the abundance of the mRNAs encoding phosphate translocator and glucosyltransferase, the GDH-synthesized RNAs up-regulated the cellulosic biomass accumulation by up to $60 \%$ [32]. The GDH-synthesized RNAs could therefore offer the research approach for investigating the influence of oxidative stress factors on fat accumulation in plants.

\section{Materials and Methods}

Peanut (Arachis hypogaea L. cv. "Valencia") seeds were sterilized with $5 \%(\mathrm{v} / \mathrm{v})$ Clorox for $10 \mathrm{~min}$, washed twice with sterile distilled water, and germinated on filter paper moistened with distilled water (control), and the solutions of oxidative compounds including $1 \%$ chitosan and $0.1 \mathrm{mM}$ ribonucleoside triphosphate (NTP) solutions as described before [33].

2.1. Fat Analyses. The fat contents of the peanut seedlings were custom analyzed by Universal Testing, Quincy, IL, USA, using standard gravimetric methods. The peanut seedlings were first oven-dried at $60^{\circ} \mathrm{C}$ to constant weight, milled (composited), and then $100 \mathrm{~g}$ per experimental treatment were sent for analysis.

2.2. Total RNA. Total RNA was extracted from the control and the treated seedlings using the acidic phenol/chloroform method [34].
2.3. Preparation of $c D N A$ Probes. The probes were the cDNAs of some of the GDH-synthesized RNA. They were prepared as follows.

\subsubsection{Purification of GDH Isoenzymes. GDH was extracted} from peanut seedlings $(30 \mathrm{~g})$ by homogenization at $4^{\circ} \mathrm{C}$ with $100 \mathrm{~mL}$ of buffer [35] containing 5 units per $\mathrm{mL}$ of each of RNase A and DNase 1 (Applied Biosystems, Foster City, CA, USA) and subjected to fractional $\left(\mathrm{NH}_{4}\right)_{2} \mathrm{SO}_{4}$ precipitation, preparative-scale isoelectric focusing (IEF; Rotofor, Bio-Rad, Hercules, CA, USA) followed by dialysis of the fractions as described before [36]. Rotofor fractions $(0.2 \mathrm{~mL})$ were purified by native $7.5 \%$ polyacrylamide gel electrophoresis (PAGE; Protean II cell, Bio-Rad) at $100 \mathrm{~V}, 20$ hours, $4^{\circ} \mathrm{C}$ to remove other proteins, and nucleic acid contaminations. GDH isoenzymes were eluted ( 30 minutes, $100 \mathrm{~V}$ ) from the electrophoresed gel with $0.05 \mathrm{M}$ solution of Tris base at subzero temperature using mini-whole gel eluter (Bio-Rad) as described before [22].

2.3.2. RNA Synthesis by GDH. RNAs were synthesized with the mini whole-gel fractions of GDH charge isomers purified from the chitosan-treated peanut seedlings. RNA synthesis was conducted in the amination direction in cocktails containing $0.87 \mathrm{mM}$ of $\mathrm{NH}_{4} \mathrm{Cl}, 3.5 \mathrm{mM}$ of $\mathrm{CaCl}_{2}, 10.0 \mathrm{mM}$ of $\alpha$-ketoglutarate $(\alpha-\mathrm{KG}), 0.23 \mathrm{mM}$ of $\mathrm{NADH}, 0.6 \mathrm{mM}$ each NTP, $5 \mathrm{U}$ of RNase inhibitor, $5 \mathrm{U}$ of DNase 1 , and $5.0 \mu \mathrm{g}$ of actinomycin $\mathrm{D}$. The reaction was started by adding $0.2 \mathrm{~mL}$ of $\mathrm{GDH}$ charge isomers containing about $4 \mu \mathrm{g}$ protein per $\mathrm{mL}$ as described before [37]. The final volume of the reaction was brought to $0.4 \mathrm{~mL}$ and $\mathrm{pH} 8$ with Tris- $\mathrm{HCl}$ solution. Reactions were incubated at $16^{\circ} \mathrm{C}$ overnight and stopped by phenol-chloroform ( $\mathrm{pH} 4.5$ ) extraction of the enzyme. The RNA was precipitated with ethanol. RNA synthesis was carried out in duplicate to verify the reproducibility of the results.

2.3.3. cDNA Synthesis, Cloning, and Characterization. cDNAs were synthesized with $2 \mu \mathrm{g}$ of peanut total RNA, and each product RNA synthesized by the GDH charge isomers, using random hexamer primer. The restriction fragment adapter ligation, PCR amplification, and sequencing gel fractionation were conducted according to the methods of Display Systems Biotech, Vista, CA, USA. Selected cDNA fragments were subcloned into pCR4-TOPO vector and transformed into TOP10 One Shot Chemically Competent Escherichia coli (Invitrogen, Carlsbad, CA, USA), followed by overnight growth on selective plates. Up to ten positive transformant colonies were picked per plate and cultured overnight in LB medium containing $50 \mu \mathrm{g} / \mathrm{mL}$ of kanamycin. Plasmid DNA was purified with a plasmid kit (Novagen, Madison, WI). The insert cDNA was sequenced with T3 and T7 primers by Genemed Synthesis, Inc (South San Francisco, CA, USA). To identify the GDH-synthesized RNAs that were homologous to mRNAs encoding LO and ACC, the cDNA sequences were used as queries to search the NCBI nucleotide-nucleotide (excluding ESTs) BLAST (blastn) and nonredundant protein translation (blastx) databases. cDNAs that displayed the highest alignment scores with mRNAs 
encoding two enzymes were selected as the probes. The NCBI BLASTN 2 Sequence alignment algorithm [38] was applied for comparison of sequence homologies.

A cDNA fragment homologous to peanut $28 \mathrm{~S}$ rRNA was also purified from the total RNA bands on the sequencing gel, to serve as probe for verifying the quality of the total RNA preparations.

2.4. Northern Blot Analysis. Equal amounts $(15 \mu \mathrm{g})$ of total RNAs from the peanut seedlings were loaded and briefly separated on $2 \%(\mathrm{w} / \mathrm{v})$ glyoxal-agarose gels and photographed to verify quality and equality of sample loading. RNA was electro-transferred with Trans-Blot SD cell (Bio-Rad, Hercules, CA) from the electrophoresed gel onto BrightstarPlus nylon membranes (Ambion, Austin, TX, USA) and immobilized to the membrane by oven heating at $85^{\circ} \mathrm{C}$ for 2 hours. To verify completeness of the electro-transfer of the total RNA, the transfer was continued on to a second Brightstar-Plus nylon membrane.

For the labeling of the cDNA probes, cDNA inserts were amplified by PCR from the corresponding plasmids (15 ng) using T7 forward and T3 reverse primers $(1 \mu \mathrm{M}$ each $),\left[\alpha^{32} \mathrm{P}\right] \mathrm{dATP}(3000 \mathrm{Ci} / \mathrm{mmol}, 10 \mathrm{mCi} / \mathrm{mL}, 2 \mu \mathrm{L})$, $\mathrm{dCTP} / \mathrm{dGTP} / \mathrm{TTP}$ mix $(50 \mathrm{mM}, 2 \mu \mathrm{L})$, Taq polymerase ( $1 \mathrm{U})$, in a final volume of $20 \mu \mathrm{L}$. Amplification was for 35 cycles $\left(95^{\circ} \mathrm{C}\right.$ for 60 seconds, $55^{\circ} \mathrm{C}$ for 30 seconds, and $72^{\circ} \mathrm{C}$ for 60 seconds), followed by final extension incubation at $72^{\circ} \mathrm{C}$ for 10 minutes. Unincorporated nucleotides were removed from the labeled cDNA inserts by chromatography through short column of sephadex G50 [39].

The first and the corresponding continuation membranes with immobilized total RNA were prehybridized with ULTRAhyb buffer (Ambion, Austin, TX, USA) at $68^{\circ} \mathrm{C}$ for 30 minutes and hybridized with ${ }^{32} \mathrm{P}$-labeled cDNA inserts as probes overnight at $68^{\circ} \mathrm{C}$. The membranes were washed $(15$ minutes, $\left.42^{\circ} \mathrm{C}\right)$ followed by another wash $\left(15\right.$ minutes, $\left.68^{\circ} \mathrm{C}\right)$ with NorthernMax low stringency wash solution (Ambion, Austin, TX, USA) to remove unbound probes. The damp membrane was exposed to X-ray film with intensifying screens at $-80^{\circ} \mathrm{C}$ for autoradiography.

The Northern band intensities were digitalized using UN-SCAN-IT gel digitizing software (Silk Scientific, Inc., Orem, Utah, USA). The digital intensities were normalized as done before with respect to GDH isomerization [20,33], but with the band of the control peanut as the base line value of 1 . For the bands of the mRNAs encoding LO and ACC, the digital intensities were rated on a scale of zero (lowest band intensity) to 10 (highest band intensity). The high molecular weight band (hypothetical protein) in the LO Northern results was excluded from the analysis.

\section{Results and Discussion}

3.1. Regulation of Peanut Fat Content by NTPs. The fat contents varied according to the treatment of the peanut seedlings (Table 1 ). The fat contents of the peanut seedlings were lower as expected compared with the seed fat contents [8]. Generally, treatments with solutions of the pyrimidine NTPs induced up to $60 \%$ more fat accumulation than the control peanut. This is because the pyrimidine NTPs are more nucleophilic than the purine NTPs, and that GDH isomerization is most strongly induced by nucleophiles $[25,28]$. An increase by $60 \%$ in the fat content of peanut will be a substantial incentive to breeders, and especially farmers to cultivate the crop for the biodiesel industry $[14,15,17]$. But the 4NTPs treatment led to the decrease of the fat content by $\sim 40 \%$ compared with the control peanut (Table 1). Based on their fat contents, the peanut seedlings fell into three groups: GTP plus CTP-, ATP-, CTP-, 3NTPs-, and UTP-treated peanuts had high fat (37.7-44.0\%) contents; control, ATP plus UTP-, and GTP-treated peanuts had medium fat contents $(27.2-32.2 \%)$; the 4 NTPs-treated peanut had low fat $(17.1 \%)$ content. Generally, fat contents tended to correlate with the trend of the GDH isoenzyme population patterns and redox gradient such that high fat contents suppressed the number of GDH isoenzymes similar to the effects of endogenous oxidative stress and nucleotide concentrations on the isomerization of the enzyme [33]. Therefore, in the oxidative stress treatments of the peanut (Table 1), the UTP solution was the upper limit because it induced the highest fat content and the lowest population of GDH isoenzymes, whereas the 4NTPs solution was the low limit because it induced the lowest fat content and the highest population of GDH isoenzymes [33]. In the enhancement of peanut cellulosic biomass by NTP solutions, the converse was the case because the purine NTP solutions induced higher biomass accumulation [32].

3.2. Isomeric RNAs. Several fragments (Table 2) of the GDHsynthesized RNAs shared short sequence homologies with the mRNAs encoding ACC and LO. Blast 2 sequence alignments [38] of those RNAs showed that they displayed stepwise overlapping plus/plus strands sequence similarities with few mismatches and gaps, and $3^{\prime}$-and/or $5^{\prime}$-nonhomologous sequences of $\sim 100$ nucleotides protruding at the ends of the alignments. This is typical of the RNAs synthesized by the GDH isoenzymes of overlapping similarities in their subunit compositions $\left(\mathrm{A}_{2} \alpha_{3} \beta, \mathrm{A}_{2} \alpha \beta_{3}, \mathrm{~A}_{4} \alpha \beta, \mathrm{A}_{3} \alpha_{2} \beta, \mathrm{A} \alpha_{4} \beta\right.$, $\mathrm{A} \alpha \beta_{4}$, etc [29]). BAST 2 Sequence alignment of probe no.1 (ACC) and probe no.3 (LO) gave six times plus/minus strands homology repeats that could explain the molecular basis of the reprogramming of the respective mRNAs. The positional orientation of the 6 subunit polypeptides constituting each isoenzyme determines the sequences of the RNA synthesized [29]. Therefore, the RNAs homologous to the mRNAs encoding ACC and LO were isomeric, like the corresponding GDH isoenzymes that synthesized them. The isomeric sequence similarities of the RNAs are evidence for the fidelity of the enzyme in the synthesis of RNA although the enzyme is a template-independent RNA polymerase.

Putative functions were assigned based on DNA database homology searches (Table 3). The databases showed that the GDH-synthesized RNA fragments that shared homology with the mRNAs encoding ACC and LO were multitarget probes in that the same RNA fragment was homologous to two or more different mRNAs. The probes for ACC were homologous to the mRNAs encoding chloroplastic and cytosolic types of the enzyme. Also, the probe for LO shared 
TABLE 1: Nucleotide-dependent reprogramming of the mRNAs encoding ACC and LO relative to the fat content of peanut seedlings. The mRNA band intensities for ACC and LO were normalized based on GDH isoenzyme population, with the control as the value of 1.0. Fat analysis was done in duplicate on composited samples and the second results were the same as the first; and with reference to their GDH isoenzyme patterns [33].

\begin{tabular}{lccc}
\hline Treatments & \% Fat & mRNA encoding & mRNA encoding \\
\hline & & ACC & LO \\
4NTPs & 17.1 & 10.0 & 5.0 \\
Control & 27.2 & 1.0 & 1.0 \\
GTP & 30.5 & $<1.0$ & 1.0 \\
ATP+UTP & 32.2 & $<1.0$ & $<1.0$ \\
GTP+CTP & 37.8 & 4.0 & $<1.0$ \\
CTP & 38.1 & 4.0 & 7.0 \\
ATP & 37.7 & $<1.0$ & 10.0 \\
3NTPs & 40.0 & 8.0 & 4.0 \\
UTP & 44.0 & 8.0 & $<1.0$ \\
\hline
\end{tabular}

TABLE 2: Nucleotide sequences of the probes. Adapter sequences flanking each probe are in bold to indicate the termini of the probe.

Probe no.1: Acetyl-Coenzyme A Carboxylase

ATTCGCCCTTATGAGTCCTGACCGAGAACGGCATTGATAGCGATGAGTCCTGACCGACAACG GCATTGATAGCGATGAGTCCTGACCGACAACGGCATTGATAGCGATGAGTCCTGACCGACAA CGGCATTGATAGCTATGAGTCCTGACCGACAACGGCATTGATAGCGATGAGTCCTGACCGGGT ACGCAGTCTACGAGAC

Probe no.2: Acetyl-Coenzyme A Carboxylase

ATTCGCCCTTTGAGTCCTGACCGAGAACGGCATTGATAGCGATGAGTCCTGACCGACAACGG CATTGATAGCGATGAGTCCTGACCGACAACGGCATTGATAGCGATGAGTCCTGACCGGCAAC GGCATTGATAGCGATGAGTCCTGACCGACAACGGCATTGATAGCGATGAGTCCTGACCGACA ACGGCATTGATAGCGATGAGTCCTGACCGGGTACGCATTCTACGAGACC

Probe no.3: Lipoxygenase

ATTCGCCCTTACTGGTCTCGTAGACTGCGTACTGGTCTCGTAGACTGCGTACTGGTCTCGTAG ACTGCGTACTGGTCTCGTAGACTGCGTACCCGGTCAGGACTCATCGCTATCAACGCCGTTCTC GGTCAGGACTCATCGCTATCGCCGATCTGGATGCGCGACTGCCCTACCTCAAGTAACACCTCC CTCCTCTGAAATGGCACGAATCCTAAGCACTGTCGTTCTCGGTCAGGACT

Probe no.4: Lipoxygenase

GGCGTTAAAGCGTTNGCGNTAGGTGGGTCTTTTAAAGTCCGTTGTGAAAAGCCCTGGGCTCNC

NCCTGGGAACTGCAGTGGATACTGGGCGACTAGAGTGTGGTANAGGGTAGCGGAATTCCTGG

TGTAGCAGTGAAATGCGTAGAGATCAGGAGGAACATCCATGGCGAAGGCAGCTACCTGGACC

AACACTGACACTGAGGCACGAAAGCGTGGGGAGCAAACAGGATTATATACCCTGGTAGTCCA

CGCCCTAAACGATGCGAACTGGATGTTGGGTGCAATTTGGCACGCAGTATCGAAGCTAACGC GTTAAGTTCGCCGCCTGGGGAGTACG

Probe no.5: 18 S rRNA

ATTCGCCCTTTGAGTCCTGACCGAGAACTGGCGATGCGGGATGAACCGGAAGTCGGGTTACG GTGCCCAACTACGCGCTAACCTAGACCCCACAAAGGGTGTTGGTCGACTTCCATGACCACCGT CCTGCTGTCTTAATCGACTTCCATGACCACCGTCCTGCTGTCTTAATCGACTTCCATGACCACC GCCCTGCTATCTTAATCGGGTACG

homology with a high molecular weight mRNA ( $~ 9000$ bases long) that encoded a hypothetical protein.

3.3. Northern Results. The results from the databases were verified by Northern analyses (Figures 1 and 2). Isomeric probe no.1 gave the best Northern result (Figure 1) for the
mRNAs encoding two types of ACC $(\sim 700$ and $\sim 8,000$ nucleotides long) because it was repeated four times with high $\mathrm{E}$ value, in the sequence of the mRNAs. Isomeric probe no.3 gave the best Northern results (Figure 2) for the mRNA encoding LO $(\sim 1,200$ nucleotides long). Probe no.4 had a low score for the mRNA encoding LO. Therefore, 
TABLe 3: Putative functions in fat metabolism assigned to the GDH-synthesized isomeric RNAs.

\begin{tabular}{lllc}
\hline Isomeric probes & Accession no. & Putative functions & E-Value \\
\hline 1 & L39267 & Acetyl-CoA carboxylase & 2.1 \\
2 & L39267 & Acetyl-CoA carboxylase & 6.5 \\
3 & AAF60270.1 & Lipoxygenase & 4.9 \\
4 & AAY87056.1 & Lipoxygenase & 9.4 \\
& AAT39222 & Hypothetical protein & 9.5 \\
5 & AF156675.2 & 18S rRNA & 6.4 \\
\hline
\end{tabular}

the isomeric sequence repeats of the GDH-synthesized RNA enabled them to serve as alternate control probes in the Northern analyses.

The duplicate syntheses of RNA by the same purified GDH isoenzymes gave identical yields and RNA fragment distribution on agarose gel electrophoresis, thus confirming reproducibility of the synthesis. The duplicate Northern analyses using each cDNA of GDH-synthesized RNA gave identical banding patterns thus confirming reproducibility of the Northern reactions. Furthermore, the experimental treatments employed were interrelated and they induced the full spectrum of the GDH isoenzymes from almost zero to the maximum of 28 isoenzymes, thus limiting the variability in sample treatment and consolidating the biological comprehensiveness of the project design [33]. The metabolic relatedness of the NTP treatments limited the variability in the chemical compositions of treatments and thus consolidated the experimental replications. On the other hand, the binomial relatedness of the GDH hexameric isoenzyme compositions [36] minimized any statistical variations in the responses of the crop's metabolism to the treatments, thus imposing a firm control on the number of experimental repeats. All total RNA preparations used were free of any degradation because the rRNA bands were present as evidenced in the photographed gels. Furthermore, Northern blots using 28S rRNA probe no.5 gave a compact band of similar intensity [40] for each total RNA irrespective of the NTP treatment of the peanut. Therefore, the total RNAs were not degraded. The second nylon membrane onto which the total RNAs were transblotted gave no bands after hybridization to labeled probes, thus confirming completeness of total RNA transfer to the first membrane.

3.4. Reprogramming of $m R N A s$. Based on their heterocyclic aromatic ring structures, the UTP is the most oxidized, and ATP is the least oxidized nucleotide. The Northern results (Figure 1) showed that the wide range of oxidative stress treatments [33] differentially regulated the abundance of the two mRNAs ( $\sim 700$ and 8,000 nucleotides long) encoding ACC. The two mRNAs were more suppressed by the purine nucleotides than by the pyrimidine nucleotides (Figure 1). But the UTP treatment suppressed the cytosolic whereas the CTP treatment suppressed the chloroplastic form. In the mixtures of nucleotides, the 4NTPs treatment enhanced the abundance of the mRNAs encoding two types of the enzyme, but the 3NTPs and the GTP plus CTP treatments enhanced the abundance of only the chloroplastic ACC. Their band intensities suggested that the mRNA encoding chloroplastic ACC was two folds more abundant than the cytosolic ACC. This could be the reason for the occurrence of the de novo synthesis of fatty acids in chloroplast [1], while the elongation of fatty acid chains occurs in the cytosol. However, the two types perform complementary roles that lead to fat accumulation. Based on the intensities of the bands (Figure 1), the peanut seedlings fell into three groups: 4NTPs-, 3NTPs-, and UTP-treated seedlings with high levels of the mRNA; GTP plus CTP-, ATP-, and CTPtreated seedlings with medium levels of the mRNA; control, ATP plus UTP-, and GTP- treated seedlings with low levels of the mRNA. The reprogramming of the mRNAs encoding ACC consequent upon the treatment of peanut with NTP solutions was therefore substantially similar to the changes in the isomerization of the GDH [33].

The trend (4NTPs $>3$ NTPs $>$ pyrimidines $>$ purines) in the band intensities of the mRNAs encoding ACC was the converse of that for the fat contents (Table 1). Therefore, there were additional molecular factors that affected the fat contents.

The mRNA encoding ACC was virtually undetectable in the ATP plus UTP-treated peanuts as compared with the control, but was up-regulated in the $3 \mathrm{NTPs}$ (ATP+GTP+UTP)treated peanut. This was a synergistic effect exerted by the nucleotide solutions on the abundance of the candidate mRNA. The synergistic effects culminated to $\sim 24 \%$ increase in fat accumulation in the 3NTPs-treated peanut. The synergistic effect also indicated an important agricultural approach that could alleviate the inhibitory influence of complex environmental factors [16] on peanut yield. The mRNA encoding ACC could serve as a molecular marker for forecasting the yield of peanut [16].

The Northern hybridization results (Figure 2) using fragment no. 3 as the probe for the mRNA $(\sim 1,200$ bases long) encoding LO showed that the control, UTP-, GTP plus CTP-, ATP plus UTP-treated seedlings had the lowest abundance of the mRNA, whilst the ATP-, 4NTPs-, and CTPtreated seedlings had the highest abundance of the mRNA, the induction of the mRNA by the pyrimidine nucleotides being intermediate between those of the purine nucleotides. Therefore, the mRNA encoding LO was reprogrammed by the oxidative stress treatments, each NTP solution exercising its specific control on the abundance of the mRNA. The high 


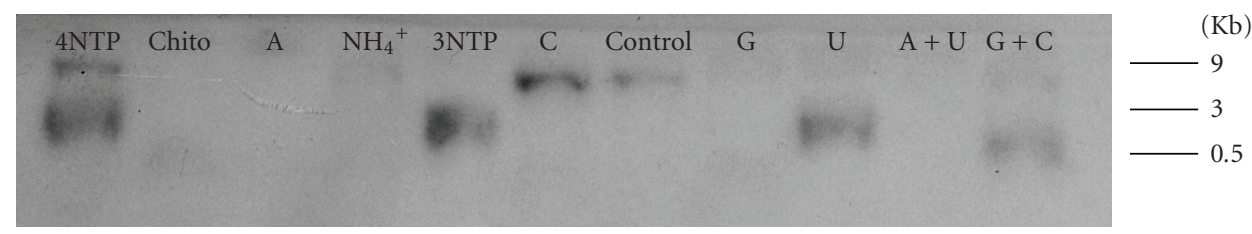

FIGURE 1: Northern hybridization with GDH-synthesized RNA no.1 as probe for acetyl-CoA carboxylase mRNA: equal amounts (15 $\mu \mathrm{g})$ of total RNAs from the control, and from $4 \mathrm{NTP}$, chitosan, ATP, $\mathrm{NH}_{4} \mathrm{Cl}$, 3NTP, CTP, GTP, UTP, ATP+UTP, and GTP+CTP-treated peanut seedlings were electrophoresed through $2 \%$ agarose gel, transblotted on to nylon membrane, followed by screening with ${ }^{32} \mathrm{P}-\mathrm{labelled} \mathrm{cDNA}$ of the probe. The membrane was washed with low stringency solution and autoradiographed. The $3 \mathrm{NTP}$ treatment was ATP+UTP+GTP solution. Northern hybridization using $28 \mathrm{~S}$ rRNA probe no.5 gave a compact band of similar intensity [40] for each total RNA irrespective of the NTP treatment thus showing that the RNA preparations were not degraded.

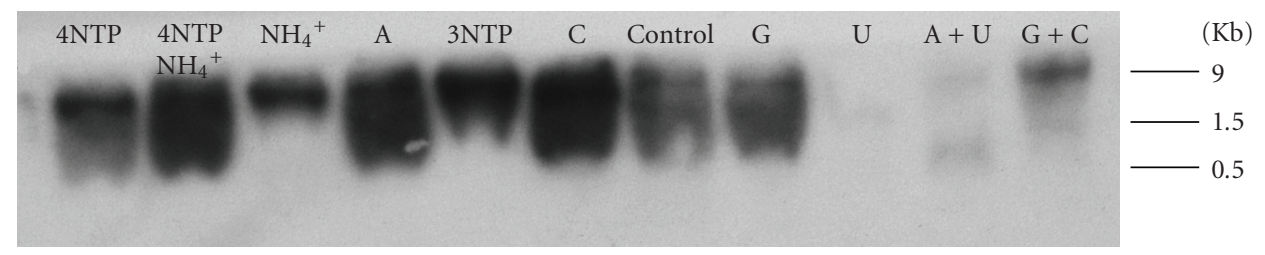

FIGURE 2: Northern hybridization with GDH-synthesized RNA no.3 as probe for lipoxygenase: equal amounts ( $15 \mu \mathrm{g})$ of total RNAs from the control, and from $4 \mathrm{NTP}, 4 \mathrm{NTP}+\mathrm{NH}_{4} \mathrm{Cl}, \mathrm{NH}_{4} \mathrm{Cl}$, ATP, 3NTP, CTP, GTP, UTP, ATP+UTP, and GTP+CTP-treated peanut seedlings were electrophoresed through $2 \%$ agarose gel, transblotted on to nylon membrane, followed by screening with ${ }^{32} \mathrm{P}-$ labelled cDNA of the probe. The membrane was washed with low stringency solution and autoradiographed. The $3 \mathrm{NTP}$ treatment was ATP+UTP+GTP solution. Northern hybridization using 28S rRNA probe no.5 gave a compact band of similar intensity [40] for each total RNA irrespective of the NTP treatment.

molecular weight band in the Northern result was that of an mRNA ( 9,000 bases long) encoding a hypothetical protein that had no effect on the mRNA for LO.

The trends in the abundance of the mRNA encoding LO compared with fat contents, and the abundance of the mRNAs encoding ACC (Table 1), however, showed that the high fat contents of the UTP-, and 3NTP-treated peanuts were characterized by very high abundance of the mRNA encoding ACC, but by low abundance of the mRNA encoding LO. Conversely, the low fat content of the 4NTPs-treated peanut was characterized by very high abundance of the mRNAs encoding ACC and LO. The medium fat contents of the control, GTP-, GTP plus CTP-, and ATP- treated peanuts were characterized by very low abundance of the mRNAs encoding ACC and LO. Therefore, LO and ACC participated jointly in the regulation of the peanut fat content. But high abundance of the mRNA encoding LO consistently led to lower fat contents, whilst low abundance led to higher fat contents. The molecular basis of the inverse relationships between the abundance of the mRNAs encoding ACC and LO on one hand, and between the high fat contents of UTPtreated and low fat contents of 4NTPs-treated peanuts on the other hand was the six-fold plus/minus strands homology between probe no.1 (ACC) and probe no.3 (LO). This was the positive evidence that the GDH-synthesized RNA reprogrammed the mRNAs encoding ACC and LO. The LOs function to dioxygenize polyunsaturated fatty acids $[2,5$, $41,42]$. The results (Table 1 ) demonstrated that in addition, the LOs dioxygenation reactions decreased the fat contents by $37 \%$ compared with the control peanut. This means that fat contents can be dramatically changed not only by breeding and genetic engineering experimentations, but also by manipulation of the GDH isomerization. Vegetable oils contain sulfur and phosphorus impurities that are bonded to the fatty acid molecules [15]. The oxygenation of the fatty acid double bonds may minimize the impurities associated with the fatty acid molecules.

Wide ranges of oxidative stress factors are not limited to the NTP solutions. What it requires is a factorial set of environmental conditions that induce the full spectrum of GDH isomerization $[20,22,23,25,26,37]$, the chemical relationships among the treatments being made to mimic the binomial relationships among the GDH isoenzyme subunits. The GDH method simplifies plant's metabolic responses to multiple environmental factors because it integrates the stimuli thereby streamlining, synchronizing, and amplifying the response reactions. Since the GDH isoenzyme pattern of the control crop was overridden and superseded by that of each treated crop [28], the comparative presentation of data (Table 1) with respect to the control was also adequate. The isomerization of GDH is initiated by the nucleophileinduced cleavage of its subunit polypeptides at the Lys residue in the active site $[43,44]$ followed by a rearrangement of the intact subunits to form a new population of hexameric isoenzymes [25], which then synthesize a new set of RNAs to regulate the abundance of mRNAs. The changes in the 
population of GDH-synthesized RNAs consequent upon changes in the oxidative status of alfalfa pasture were staggering [20]. Similarly, changes in the abundance of mRNAs consequent upon changes in the population of GDH-synthesized RNAs were also staggering (Figures 1 and 2). Finally, changes in the concentrations of metabolites consequent upon changes in the abundance of mRNAs were also substantial (Table 1). The positional orientations of the GDH subunit polypeptides in each isoenzyme determine the sequences of the RNA synthesized and the fidelity of the enzyme in the synthetic reaction. Furthermore, the binomial statistical arrangement of the six subunit polypeptides in each isoenzyme [36] assures the synthesis of an almost infinite permutation of short RNA sequences that would be homologous to numerous mRNA under the prevalent oxidative state. Therefore, the templateless synthesis of RNA by GDH is a special feature conferred by the prevalent oxidative status of the crop or pasture on the positionality of the subunit polypeptides within each hexameric isoenzyme. The integration of the prevalent oxidative status by the GDH isomerization [28] followed by RNA synthesis is the basis of the reprogramming of mRNA abundance.

The mechanism by which the LOs affected the fat content was not investigated but could be the result of the oxygenation of the polyunsaturated fatty acids to hydroxyl fatty acids followed by their conversion to other metabolic products [7]. The fatty acid and flavonoid profiles of the peanuts could illuminate the mechanism. Most of the research on the LOs has focused on the pharmacologically useful products of the oxygenation reaction [45]. The ability of the LO to alter the quantity of fat content could offer alternative technological method for improving the purity of vegetable oil for biodiesel production.

\section{Conclusion}

The regulation of fat accumulation in peanut seedlings by NTP solutions was studied. The abundance of the mRNAs encoding ACC and LO was studied using Northern analysis. The GDH isomerization was fully induced by the wide array of NTP solutions. The Northern probes were the GDH-synthesized RNAs that displayed sequence homology with the mRNAs encoding two candidate enzymes. Fat accumulation increased from about $27 \%$ in the control peanut to about $44 \%$ in the UTP-treated peanut seedlings. The increases in the levels of the mRNA encoding ACC were inversely related to the fat accumulated. Those NTP solutions (UTP, 3NTPs) that up-regulated the mRNA encoding ACC but down-regulated the mRNA encoding LO up-regulated the fat accumulation by about $48 \%$ compared with the control. Conversely, the NTP solution (4NTPs) that up-regulated the mRNAs encoding ACC and LO down-regulated the fat accumulation by about $60 \%$. The cDNA probes used in the Northern analyses displayed six-fold plus/minus strands sequence homologies. Therefore, LO, ACC, and the GDHsynthesized RNAs participated directly in the regulation of fat accumulation in the seedlings. These results could be useful in the environmental manipulation of peanuts to produce specific quality fat for the food and bioenergy industries.

\section{References}

[1] S. Reverdatto, V. Beilinson, and N. C. Nielsen, "A multisubunit acetyl coenzyme a carboxylase from soybean," Plant Physiology, vol. 119, no. 3, pp. 961-978, 1999.

[2] M. J. Nelson and S. P. Seitz, "The structure and function of lipoxygenase," Current Opinion in Structural Biology, vol. 4, no. 6, pp. 878-884, 1994.

[3] T. Konishi, K. Shinohara, K. Yamada, and Y. Sasaki, "AcetylCoA carboxylase in higher plants: most plants other than gramineae have both the prokaryotic and the eukaryotic forms of this enzyme," Plant and Cell Physiology, vol. 37, no. 2, pp. 117-122, 1996.

[4] Y. Sasaki, T. Konishi, and Y. Nagano, "The compartmentation of acetyl-coenzyme A carboxylase in plants," Plant Physiology, vol. 108, no. 2, pp. 445-449, 1995.

[5] H. W. Gardner, "Recent investigations into the lipoxygenase pathway of plants," Biochimica et Biophysica Acta, vol. 1084, no. 3, pp. 221-239, 1991.

[6] S. T. Crooke and A. Wong, Eds., Lipoxygenases and Their Products, Academic Press, San Diego, Calif, USA, 1991.

[7] J. Van der Zee, T. E. Eling, and R. P. Mason, "Formation of free radical metabolites in the reaction between soybean lipoxygenase and its inhibitors. An ESR study," Biochemistry, vol. 28, no. 21, pp. 8363-8367, 1989.

[8] E. M. Ahmed and C. T. Young, "Composition, quality, and flavor of peanuts," in Peanut Science and Technology, H. E. Pattee and C. T. Young, Eds., pp. 656-688, American Peanut Research and Education Society, Yoakum, Tex, USA, 1982.

[9] G. Sempore and J. Bezard, "Qualitative and quantitative analysis of peanut oil triacylglycerols by reversed-phase liquid chromatography," Journal of Chromatography A, vol. 366, pp. 261-282, 1986.

[10] A. Belkebir, R. D. Paepe, A. Trémolières, F. Aïd, and G. Benhassaine-Kesri, "Sethoxydim affects lipid synthesis and acetyl-CoA carboxylase activity in soybean," Journal of Experimental Botany, vol. 57, no. 14, pp. 3553-3562, 2006.

[11] R. Delourme, C. Falentin, V. Huteau, et al., "Genetic control of oil content in oilseed rape (Brassica napus L.)," Theoretical and Applied Genetics, vol. 113, no. 7, pp. 1331-1345, 2006.

[12] C. A. McCartney, R. Scarth, P. B. E. McVetty, and J. K. Daun, "Genotypic and environmental effects on saturated fatty acid concentration of canola grown in Manitoba," Canadian Journal of Plant Science, vol. 84, no. 3, pp. 749-756, 2004.

[13] A. Schierholt and H. C. Becker, "Environmental variability and heritability of high oleic acid content in winter oilseed rape," Plant Breeding, vol. 120, no. 1, pp. 63-66, 2001.

[14] G. Shumaker, J. C. McKissick, and N. Smith, "Economics of peanuts for biodiesel production. Center for Agribusiness and Economic Development College of Agricultural and Environmental Sciences," Tech. Rep. CR-07-04, The University of Georgia, Athens, Ga, USA, 2007.

[15] M. Cassap, "Ensuring high-quality biodiesel," Laboratory Equipment, vol. 46, pp. 29-31, 2009.

[16] P. Kaur and S. S. Hundal, "Forecasting growth and yield of groundnut (Arachis hypogaea) with a dynamic simulation model 'PNUTGRO' under Punjab conditions," Journal of Agricultural Science, vol. 133, no. 2, pp. 167-173, 1999.

[17] A. J. Norden, O. D. Smith, and D. W. Gorbet, "Breeding of the cultivated peanut," in Peanut Science and Technology, H. E. Pattee and C. T. Young, Eds., pp. 95-122, American Peanut Research and Education Society, Yoakum, Tex, USA, 1982. 
[18] G. O. Osuji and W. C. Madu, "Regulation of sweetpotato growth and differentiation by glutamate dehydrogenase," Canadian Journal of Botany, vol. 75, no. 7, pp. 1070-1078, 1997.

[19] R. Ameziane, K. Bernhard, and D. Lightfoot, "Expression of the bacterial $g d h A$ gene encoding a NADPH glutamate dehydrogenase in tobacco affects plant growth and development," Plant and Soil, vol. 221, no. 1, pp. 47-57, 2000.

[20] G. Osuji, V. Haby, and D. Chessman, "Metabolic responses induced by serial harvesting of alfalfa pasture established on amended acid soil," Communications in Soil Science and Plant Analysis, vol. 37, no. 9-10, pp. 1281-1301, 2006.

[21] H. S. Srivastava and R. P. Singh, "Role and regulation of L-glutamate dehydrogenase activity in higher plants," Phytochemistry, vol. 26, no. 3, pp. 597-610, 1987.

[22] G. O. Osuji, V. A. Haby, D. J. Chessman, and A. T. Leonard, "Responses of the fructose-1,6-bisphosphatase and glutamate dehydrogenase activities of alfalfa to boron, gypsum, and limestone amendments of soil," Photosynthetica, vol. 42, no. 2, pp. 307-312, 2004.

[23] G. O. Osuji, V. A. Haby, A. Beyene, W. C. Madu, and A. S. Mangaroo, "The isomerization of glutamate dehydrogenase in response to lead toxicity in maize," Biologia Plantarum, vol. 40, no. 3, pp. 389-398, 1997.

[24] G. O. Osuji, "Peanut glutamate dehydrogenase: a target site of herbicide action," in Plant Nutrition for Sustainable Food Production and Environment, T. Ando, K. Fujita, T. Mae, H. Matsumoto, S. Mori, and J. Sekiya, Eds., pp. 845-850, Kluwer Academic Publishers, Tokyo, Japan, 1997.

[25] G. O. Osuji, C. Braithwaite, R. Pointer, and J. Reyes, "Pesticide inactivation of peanut glutamate dehydrogenase: biochemical basis of the enzyme's isomerization," Journal of Agricultural and Food Chemistry, vol. 47, no. 8, pp. 3345-3351, 1999.

[26] G. O. Osuji, J. C. Reyes, and A. S. Mangaroo, "Glutamate dehydrogenase isomerization: a simple method for diagnosing nitrogen, phosphorus, and potassium sufficiency in maize (Zea mays L.)," Journal of Agricultural and Food Chemistry, vol. 46, no. 6, pp. 2395-2401, 1998.

[27] G. O. Osuji and W. C. Madu, "Ammonium ion salvage by glutamate dehydrogenase during defence response in maize," Phytochemistry, vol. 42, no. 6, pp. 1491-1498, 1996.

[28] G. O. Osuji and C. Braithwaite, "Signaling by glutamate dehydrogenase in response to pesticide treatment and nitrogen fertilization of peanut (Arachis hypogaea L.)," Journal of Agricultural and Food Chemistry, vol. 47, no. 8, pp. 3332-3344, 1999.

[29] G. O. Osuji and T. Brown, "Role of the RNAs synthesized by glutamate dehydrogenase in the coordinate regulation of metabolic processes," ICFAI Journal of Biotechnology, vol. 1, no. 3, pp. 37-48, 2007.

[30] M. R. Broadley and P. J. White, Plant Nutritional Genomics, CRC Press, Boca Raton, Fla, USA, 2005.

[31] A. Hall, Crop Responses to Environment, CRC Press, London, UK, 2001.

[32] G. O. Osuji and T. Brown, "Environment-wide reprogramming of the mRNAs encoding glucosyltransferase and phosphate translocator in relation to cellulosic biomass accumulation," ICFAI Journal of Biotechnology, vol. 1, no. 4, pp. 35-47, 2007.

[33] G. O. Osuji, W. C. Madu, C. Braithwaite, et al., "Nucleotidedependent isomerization of glutamate dehydrogenase in relation to total RNA contents of peanut," Biologia Plantarum, vol. 47, no. 2, pp. 195-202, 2004.
[34] D. Grierson, A. Slater, J. Speirs, and G. A. Tucker, "The appearance of polygalacturonase mRNA in tomatoes: one of a series of changes in gene expression during development and ripening," Planta, vol. 163, no. 2, pp. 263-271, 1985.

[35] V. M. Loyola-Vargas and E. S. De Jimenez, "Differential role of glutamate dehydrogenase in nitrogen metabolism of maize tissues," Plant Physiology, vol. 76, no. 2, pp. 536-540, 1984.

[36] G. O. Osuji and W. C. Madu, "Ammonium ion-dependent isomerization of glutamate dehydrogenase in relation to glutamate synthesis in maize," Phytochemistry, vol. 39, no. 3, pp. 495-503, 1995.

[37] G. O. Osuji, J. Konan, and G. M'Mbijjewe, "RNA synthetic activity of glutamate dehydrogenase: determination of enzyme purity, RNA characteristics, and deamination/amination ratio," Applied Biochemistry and Biotechnology, vol. 119, no. 3, pp. 209-228, 2004.

[38] T. A. Tatusova and T. L. Madden, "BLAST 2 SEQUENCES, a new tool for comparing protein and nucleotide sequences," FEMS Microbiology Letters, vol. 174, no. 2, pp. 247-250, 1999.

[39] K. Struhl, "Separating radioactively labeled DNA from unincorporated dNTP precursors by column chromatography," in Short Protocols in Molecular Biology, F. M. Ausbel, R. Brent, R. E. Kingston, et al., Eds., pp. 3-18-3-19, Greene Publishing Associates and John Wiley \& Sons, New York, NY, USA, 1992.

[40] G. O. Osuji, T. K. Brown, and S. M. South, "Discovery of the RNA synthetic activity of glutamate dehydrogenase and its application in drug metabolism research," The Open Drug Metabolism Journal, vol. 2, pp. 1-13, 2008.

[41] B. J. Gaffney, D. V. Mavrophilipos, and K. S. Doctor, "Access of ligands to the ferric center in lipoxygenase-1," Biophysical Journal, vol. 64, no. 3, pp. 773-783, 1993.

[42] F. Clemens, G. Drutkowski, M. Wiese, and P. Frohberg, "The inactivation of lipoxygenase-1 from soybeans by amidrazones," Biochimica et Biophysica Acta, vol. 1549, no. 1, pp. 8898, 2001.

[43] A. Brown, J. M. Culver, and H. F. Fisher, "Mechanism of inactivation of L-glutamate dehydrogenase by pyridoxal and pyridoxal phosphate," Biochemistry, vol. 12, no. 22, pp. 43674373, 1973.

[44] G. O. Osuji, A. S. Mangaroo, and P. S. Roberts, "In vitro isomerization of glutamate dehydrogenase in relation to phytosequesteration of lead," SAAS Bulletin, Biochemistry and Biotechnology, vol. 14, pp. 60-72, 2001.

[45] C. M. Chinnici, Y. Yao, T. Ding, C. D. Funk, and D. Praticò, "Absence of 12/15 lipoxygenase reduces brain oxidative stress in apolipoprotein E-deficient mice," American Journal of Pathology, vol. 167, no. 5, pp. 1371-1377, 2005. 

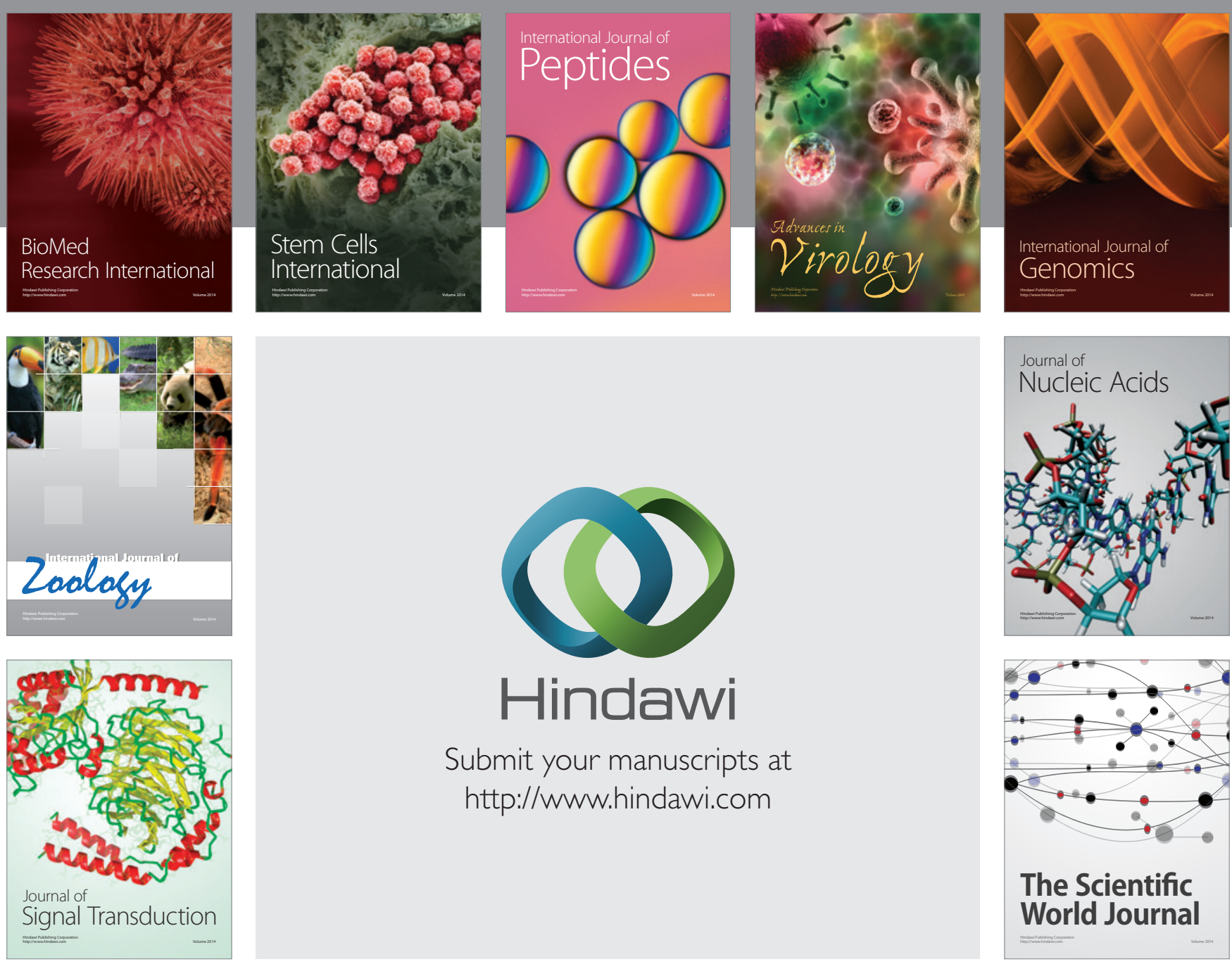

Submit your manuscripts at

http://www.hindawi.com
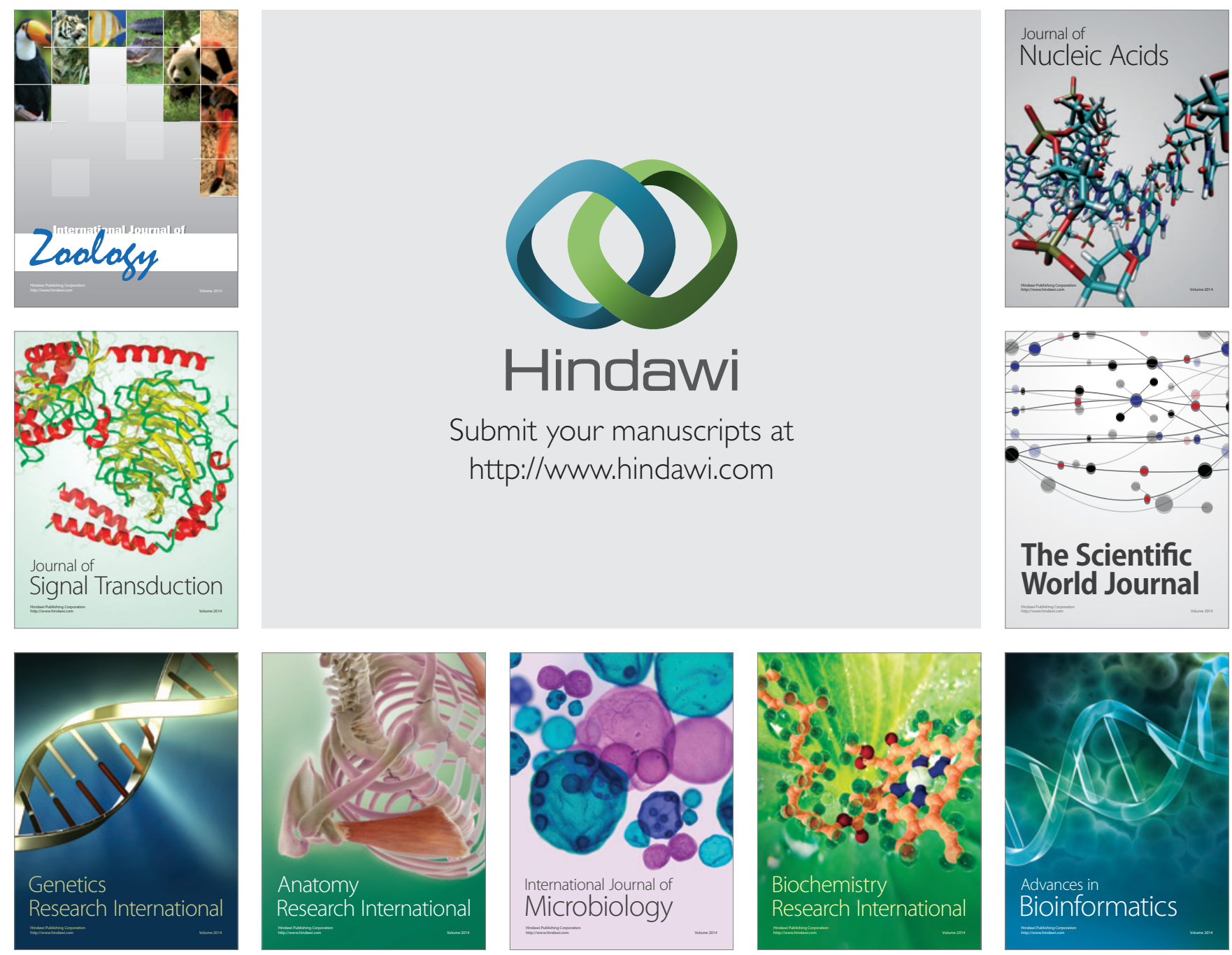

The Scientific World Journal
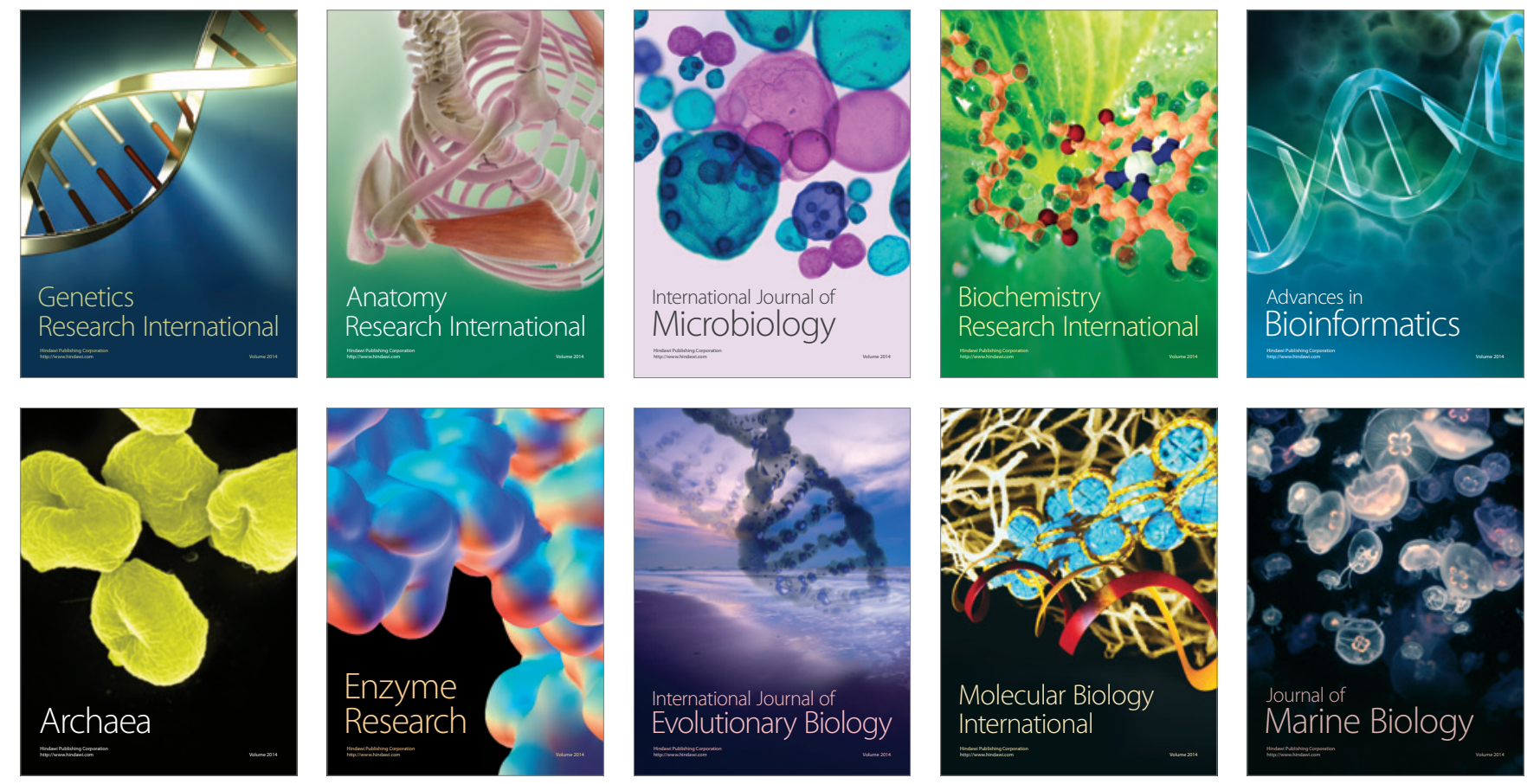